\title{
Original
}

\section{Role of Heparanase in the Release of Heparan Sulphate Binding Growth Factors in Odontogenic Tumors}

\author{
Phuu Pwint Han ${ }^{1,2)}$, Hitoshi Nagatsuka²), Ryo Tamamura'), Naoki Katase ${ }^{2)}$, Lefeuvre Mathieu Bernard ${ }^{2)}$, \\ Hailong $\mathrm{Hu}^{2)}$, Shin Takagi ${ }^{3)}$, Nobuhisa Ishida ${ }^{3)}$, Keisuke Nakano(), Toshiyuki Kawakami ${ }^{4)}$ and Mehmet Gunduz ${ }^{2)}$ \\ 1) Orofacial Pain and Oral Medicine Center, Division of Diagnostic Sciences, School of Dentistry, University of Southern California, \\ Los Angeles, USA. \\ 2) Department of Oral Pathology and Medicine, Graduate School of Medicine, Dentistry and Pharmaceutical Sciences, Okayama \\ University, Okayama, Japan. \\ 3) Department of Oral Surgery I, Graduate School of Medicine, Dentistry and Pharmaceutical Sciences, Okayama University, Okayama, \\ Japan. \\ ${ }^{4)}$ Hard tissue Pathological Unit, Matsumoto Dental University Graduate of Oral Medicine, Shiojiiri,399-0781 Japan \\ (Accepted for publication, Junuary 18, 2007)
}

\begin{abstract}
Immunolocalization of heparan sulphate(HS) and heparanase was evaluated in benign and malignant odontogenic tumors in order to know whether these molecules have potential roles in odontogenic tumorigenesis. Archival formalin-fixed, paraffin-embedded tissues of 6 human tooth germs, 7 adenomatoid odontogenic tumors, 10 ameloblastic fibromas, 20 ameloblastomas and 4 ameloblastic carcinomas were subjected to immunohistochemical staining using antibodies to HS, heparanase and BMP-4. HS was ubiquitously localized in tooth germ but heparanase and BMP-4 were observed in limited areas only. In benign epithelial tumors such as ameloblastoma and adenomatoid odontogenic tumor, these molecules were localized in neoplastic epithelium but in ameloblastic fibroma, positive reactions were observed in both epithelial and mesenchymal cells. Stromal localization of HS and BMP-4 accompanied by intense immunoexpression of epithelial heparanase was observed in ameloblastic carcinoma,and this may represent the malignant progression of ameloblastoma to ameloblastic carcinoma. Stronger intensity and more diffuse localization of heparanase in odontogenic tumors compare to that of human tooth germ was the most significant finding. Taken together, the results inferred that heparanase may be responsible for the growth and progression of odontogenic tumors by modulating the availability and function of HS binding growth factors and the derangement in immunoexpression and localization of HS and heparanase molecules may have important roles in progression of malignant odontogenic tumors.
\end{abstract}

Key words: Heparanase, Heparan sulphate, BMP-4, Odontogenic tumors, Tooth germ, Immunohistochemistry

\section{Introduction}

Heparan sulphate proteoglycans (HSPG) constitute a group of ubiquitous extracellular matrix macromolecules and are composed of a core protein and covalently linked heparan sulphate (HS) sugar chains ${ }^{1)}$. Although HSPG play critical functions in cell-cell and cell-matrix interactions through the core proteins, their HS chains confer most of the biological functions ${ }^{1,2)}$. The negatively charged HS chains can bind and sequester numerous heparin/HS binding molecules ranging from growth factors, cytokines and cell adhesion molecules thereby protecting these tethered molecules from proteolytic cleavage. HS chains also take part in the important cellular events conferred by these tethered molecules

Corresponding to Dr. Phuu Pwint Han, Department of Oral Pathology and Medicine, Graduate School of Medicine, Dentistry and Pharmaceutical Sciences, Okayama University, 2-5-1 Shikata Cho, Okayama 700-8525, Japan. Tel: 81-(86)-235-6652, Fax: 81-(86)-235-6654, Email: phuupwinthan@gmail.com and have influence on various developmental and pathological processes $^{2,3)}$.

Heparanase is a mammalian endo-â-glucuronidase enzyme capable of selectively degrading HS chains at specific intrachain sites. Although there are several distinct HS degrading endoglycosidases, cloning of a single gene and subsequent biochemical characterization suggests that mammalian cells primarily express a single dominant heparanase ${ }^{4)}$. The degraded HS chains are biologically active causing the release of HS bound molecules in large quantities for respective functions ${ }^{5}$. Furthermore, HSPG are integral components of extracellular matrix (ECM) and basement membrane (BM) and the cleavage of HS chains by heparanase can cause profound decrease in ECM and BM integrity. These were exemplified in the increased expression of heparanase in a number of human malignancies which correlated with disease progression and metastasis ${ }^{4,5}$. Apart 


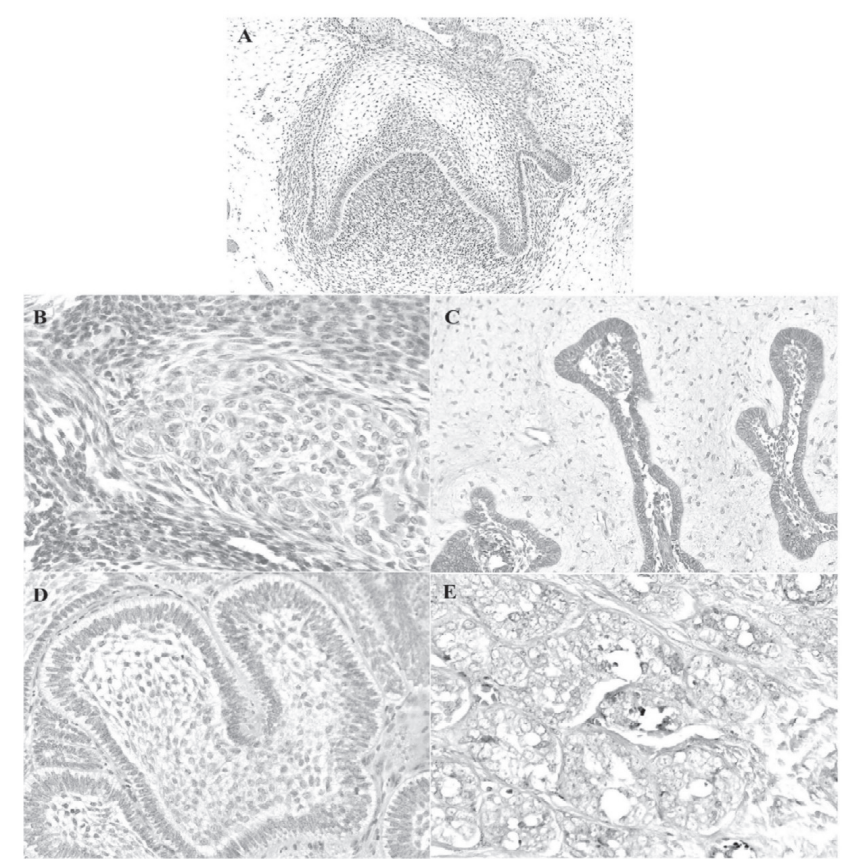

Figure 1. HE sections of human tooth germ and odontogenic tumors Late cap or early bell stage human tooth germ with columnar preameloblastic cells of inner enamel epithelium (A). AOT showing the neoplastic odontogenic epithelium forming solid tumor nests, whorl-like structures and duct-like structures with eosinophilic extracellular matrices (B). Scattered odontogenic epithelial islands within dental papilla-like ectomesenchymal tissue characterized the histologic features of ameloblastic fibroma (C). Follicular ameloblastoma was identified by nests or islands of odontogenic epithelium with peripheral columnar ameloblastlike cells and central stellate reticulum-like cells, and mature fibrous stroma (D). HE of ameloblastic carcinoma showed nests of cancer cells with increased nuclear cytoplasmic ratio and prominent nuclei (E).

from its enzymatic activity, heparanase is also reported to have cell adhesion function through cytoskeletal elements ${ }^{6)}$ and angiogenic function through direct inductive effect on COX-2 and VEGF expression ${ }^{7,8)}$. However, heparanase is not only essential for tumor progression but also important for physiologic conditions such as morphogenesis, tissue repair and remodeling, immune surveillance and HS turn over ${ }^{5}$.

Mammalian odontogenesis is a complex process involving the sequential and reciprocal inductive interactions between odontogenic epithelium and ectomesenchyme ${ }^{9)}$. These interactions involve complex networks of various signaling molecules, growth factors and their receptors in which most are bound to $\mathrm{HS}^{9,10)}$. On the other hand, odontogenic tumors constitute a group of heterogeneous lesions ranging from hamartomatous proliferations and benign neoplasms to overt malignant tumors with metastatic potential. They are derived from epithelial, ectomesenchymal and/ or mesenchymal elements of the tooth-forming apparatus and are classified according to the biological character (benign or malignant) and their neoplastic components ${ }^{11)}$. It has been reported that HSPG are localized in mouse molar tooth germ and in some odontogenic tumors ${ }^{12,13,14)}$ and heparanase in ameloblastoma ${ }^{15)}$ by

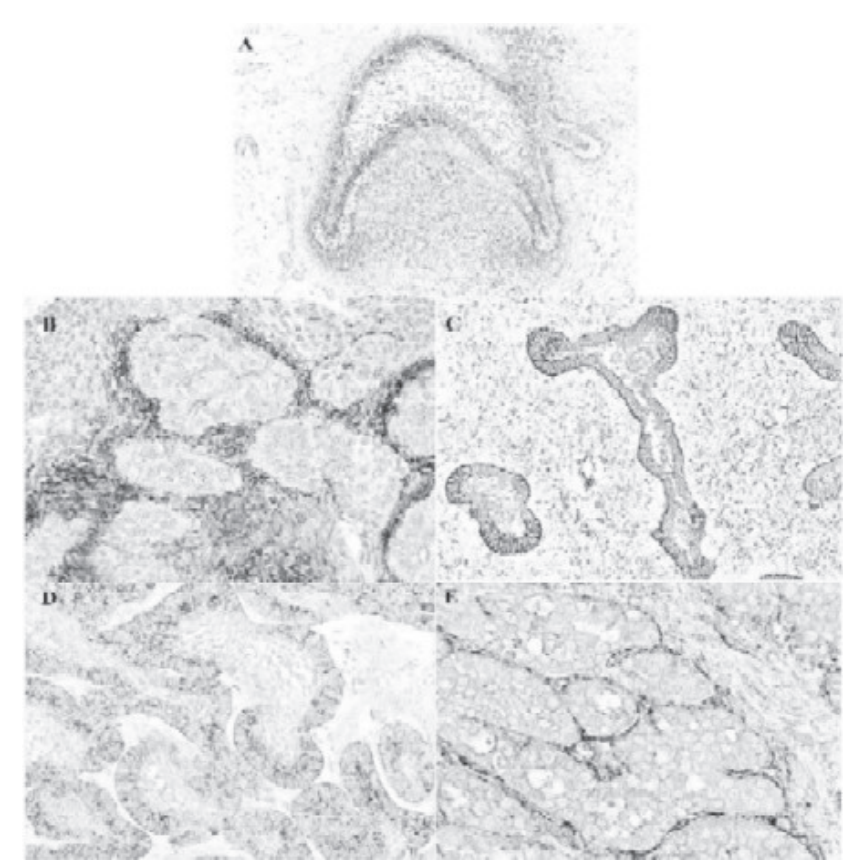

Figure 2. Distribution of HS in human tooth germ and odontogenic tumors HS was ubiquitously localized in the tooth germ with strongest intensity in stratum intermedium of the enamel organ and in the dental papilla cells (A). Strong HS staining was noted in dark, cuboidal cells surrounding the whorls and solid nests of AOT, and weak expression was noted in eosinophilic matrices as well as in the luminal surfaces of duct-like structures (B). The epithelial cells as well as the dental papilla-like cells ectomesenchymal cells of ameloblastic fibroma were reactive to HS. HS immunoreactivity was accentuated in the budding areas of the epithelial tumor nests, at the BM surrounding epithelial nests and at the cellular processes of mesenchymal cells (C). In ameloblastoma, strong and clear extracellular staining was noted in peripheral ameloblast-like cells, some suprabasal cells and at the BM as membranous and linear patterns but not in the central stellate-like cells (D). HS was not detected in the cancer cells of ameloblastic carcinoma but was strongly localized in the stromal tissues adjacent to cancer nests (E).

independent studies. Most of the HS bound molecules important for odontogenesis have also been identified in odontogenic tumors $^{16)}$.

For these reasons, it is possible that HS and heparanase may have functional roles in odontogenesis and odontogenic tumors. However, their localization patterns, relation and putative functional roles in human tooth germ and in different types of odontogenic tumors have not yet been explored. This study aimed to determine the localization of HS and heparanase in human tooth germ and in odontogenic tumors and to analyze the role of these molecules in relation to the biological character, differentiation and progression of odontogenic tumors. Among the various heparin-binding molecules, BMP-4, which is known to have important functional roles in odontogenesis ${ }^{17)}$ and in odontogenic tumors $^{18)}$ was used as an example.

\section{Materials and Methods}

Embryonic human tooth germs (in the cap/early bell stage) were 
Han Phuu Pwint et al: Role of Heparanase in Odontogenic Tumors

Table I Immunolocalization of HS, Heparanase and BMP-4 in human tooth germ

\begin{tabular}{|ccccc|ccccc|ccccc|}
\hline \multicolumn{5}{|c|}{ HS } & \multicolumn{5}{|c|}{ Heparanase } & \multicolumn{5}{c|}{ BMP-4 } \\
\hline IEE & SI & OEE & DL & DP & IEE & SI & OEE & DL & DP & IEE & SI & OEE & DL & DP \\
\hline \pm & + & \pm & + & + & \pm & \pm & \pm & \pm & \pm & - & \pm & \pm & \pm & - \\
\hline
\end{tabular}

IEE - Inner Enamel Epithelium, SI - Stratum Intermedium, OEE - Outer Enamel Epithelium, DL - Dental Lamina, DP - Dental Papilla no staining, \pm weak or focal, + strong

Table II The distribution patterns of HS, heparanase and BMP-4 in odontogenic tumors

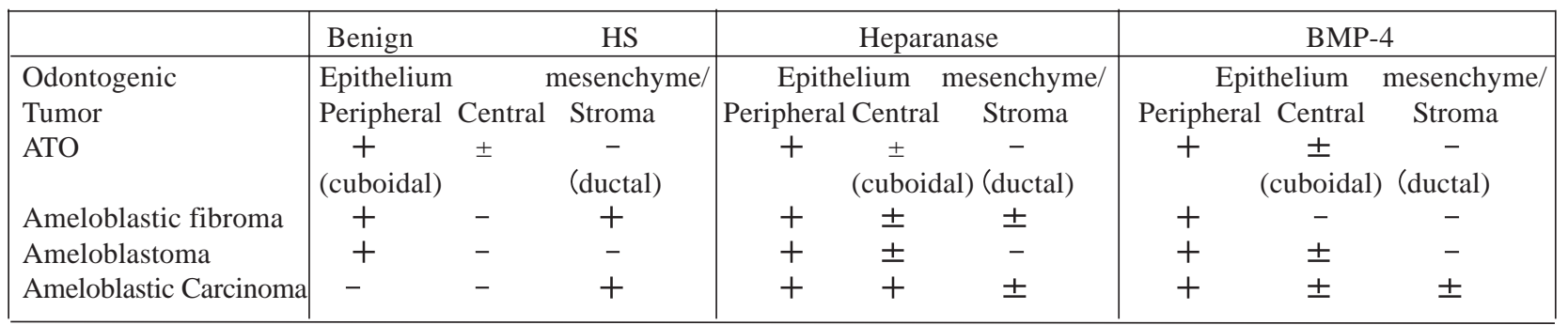

- no staining, \pm weak or focal, + strong

kindly provided by Professor Shigehisa Yamamoto from the Department of Oral Histology, Ohu University Dental School and the tumor samples studied were from the surgical pathology section of the Department of Oral Pathology and Medicine, Graduate School of Medicine, Dentistry and Pharmaceutical Sciences, Okayama University. Archival formalin-fixed, paraffinembedded tissue blocks of 6 human tooth germs, 7 adenomatoid odontogenic tumors, 10 ameloblastic fibromas, 20 ameloblastomas and 4 ameloblastic carcinomas were selected. New serial tissue sections cut at $4 \mu \mathrm{m}$ were used for routine H\&E staining and immunohistochemical staining. Histological diagnoses of the tumor entities were made and confirmed according to the 2005 World Health Organization (WHO) classification of odontogenic tumors ${ }^{11)}$ (Fig. 1).

\section{Antibodies}

Mouse monoclonal antibody 10E4 (Seikagaku Corp., Japan) that recognizes common $\mathrm{N}$-sulfated and $\mathrm{N}$-acetylated glucosamine sugar units in the native HS sugar chains regardless of the core protein structure and localization was used in this study ${ }^{19)}$. For detection of heparanase and BMP-4, mouse monoclonal antibody to human heparanase ${ }^{7,16)}$ and goat polyclonal antibody to human BMP-4 (Santa Cruz Biotech Inc., USA) were used. Antibody detection was achieved by mouse IgG ABC kit or goat IgG ABC kit (Vectastain Elite ABC kits, Vector Laboratory, USA) accordingly and the immunoreaction was visualized by 3,3'diaminobenzidine tetrahydrochloride (Hitstofine DAB substrate, Nichirei, Japan).

\section{Immunohistochemistry}

The deparaffinized sections were treated with methanol containing $0.3 \% \mathrm{H}_{2} \mathrm{O}_{2}$ for 30 min to block endogenous peroxidase activity. The tissue sections were either microwaved in $10 \mathrm{mM}$ citrate buffer solution pH 6.0 (heparanase and BMP-4) or treated with $0.1 \%$ trypsin/ $\mathrm{CaCl}_{2}$ solution for $5-15$ min (10E4 antibody) for antigen retrieval. After blocking of non-specific reactivity with normal serum (ABC kits) for $20 \mathrm{~min}$ at room temperature, the sections were incubated overnight with respective primary antibodies (10E4-1:500, heparanase-1:1500, BMP-4-1:100) at $4{ }^{\circ} \mathrm{C}$. The tagging of primary antibodies was achieved by subsequent application of biotinylated anti-IgG antibodies and avidin-biotin complexes (ABC kits). Visualization of immunohistochemical reaction was performed by developing the enzyme complex with DAB solution containing $0.01 \% \mathrm{H}_{2} \mathrm{O}_{2}$ and the nuclei were counterstained with Mayer's hematoxylin. For negative control, sections were incubated with omission of the primary antibody under the same protocol. Positive control sections were included for each antibody and internal staining controls, if present in the specimen, were checked for appropriate reactions with each antibody. In each lesion, assessment of immunostaining was based on granular staining at the cell surface, in cytoplasm and/or nucleus of tumor cells and stromal cells, and also at intercellular spaces and BMs.

\section{Results}

Immunohistochemical reactivities for HS, heparanase and BMP-4 are summarized in Table I and II. In general, HS was primarily observed extracellularly on cell membranes, in intercellular spaces and at the BMs. On the other hand, heparanase and BMP-4 were mostly detected in the cytoplasm; BMP-4 with more limited staining.

\section{Localization of Heparan Sulphate (Fig. 2)}

HS chains were localized most prominently in stratum intermedium of enamel organ. The palisaded pre-ameloblasts of the inner enamel epithelium showed cytoplasmic and membranous 




Figure 3.Immunolocalization of heparanase in human tooth germ and odontogenic tumors

Heparanase was limited to some epithelial cells of the enamel organ especially at the growing cervical loop areas and the dental lamina of the permanent tooth. Random nuclear staining was noted in some epithelial cells of the enamel organ and more commonly in the mesenchymal cells of dental papilla (A). Diffuse staining was noted in tumor cells of AOT with the strongest intensity in dark, cuboidal cells forming cribriform structures at the periphery of the solid tumor nests and whorl-like structures (B). Although the epithelial cells at the budding areas showed stronger reaction, heparanase was noted in the epithelial cells as well as the mesenchymal cells of ameloblastic fibroma (C). Strong cytoplasmic staining was observed in the peripheral ameloblast-like cells of ameloblastoma but no significant staining was noted in central stellatelike cells and in the mature fibrous connective tissue (D). Very intense and diffuse immunoexpression was observed in all cancer cells of ameloblastic carcinoma in contrast to strong staining limited to peripheral cells of benign ameloblastoma (E).

staining. Dental BM showed linear staining to HS and the dental papilla cells also showed intense membranous as well as extracellular staining to HS (Fig. 2, A). In the case of AOT, HS was relatively limited and strongly localized at the surfaces of dark cuboidal cells surrounding the whorls and solid epithelial nests, resembling partitions in between tumor nests (Fig. 2, B). In addition, well-delineated staining to HS was observed in luminal surfaces of some duct-like structures. The eosinophilic matrix in the lumen of some of these whorls and duct-like structures were also partially positive. Ameloblastic fibroma is a mixed odontogenic tumor comprising both epithelial and ectomesenchymal components of odontogenic apparatus. Generally, ameloblastic fibroma showed similar immuno expression pattern of HS as in the tooth germ. Epithelial nests and strands of ameloblastic fibroma showed staining to HS especially at the budding areas of the nests but BM confinement was more prominent than that of the tooth germ. HS staining was also observed in ectomesenchymal cells of ameloblastic fibroma (Fig. 2, C). HS was observed clearly on

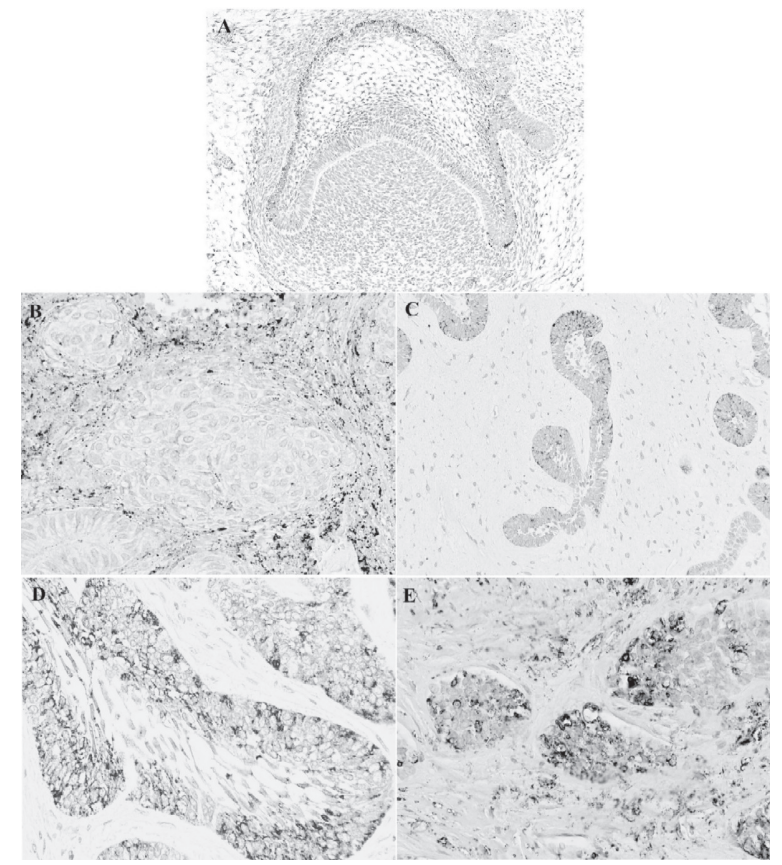

Figure 4. Immunoexpression of BMP-4 in human tooth germ and odontogenic tumors

Epithelial cells of the outer enamel epithelium and growing cervical loops showed positive reaction to BMP-4 but no apparent staining was noted in the inner enamel epithelium and the dental mesenchyme of the tooth germ (A). The dark cuboidal cells between the whorls and solid tumor nests of AOT showed the strongest immunostaining (B). BMP-4 was localized in the columnar epithelial cells of ameloblastic fibroma (C) and ameloblastoma (D) with more prominent staining in the latter. Random but significant staining was noted in cancer cells as well as the surrounding stroma of ameloblastic carcinoma (E).

the cell surfaces of peripheral basal cells and also in intercellular spaces of some suprabasal cells of ameloblastoma but almost negative in the central stellate cells (Fig. 2, D). The stromal cells of ameloblastoma did not show significant immunoreactivity to HS unlike the ectomesenchymal cells of ameloblastic fibroma. Contrary to ameloblastoma, cancer cells of ameloblastic carcinoma were not reactive to HS. Instead, a strong immunostaining was observed in the stromal compartment especially at the immediate vicinity of cancer nests (Fig. 2, E). Note that the BMs surrounding the cancer nests were unclear.

\section{Localization of Heparanase (Fig. 3)}

In human tooth germ, heparanase staining was much limited to the epithelial cells of the growing cervical loops and the dental lamina cells of permanent tooth. Random but clear nuclear staining to heparanase was also observed in epithelial as well as in some mesenchymal cells of the tooth germ (Fig. 3, A). Interestingly, diffuse cytoplasmic staining to heparanase was observed in epithelial cells of AOT with stronger staining in dark cuboidal cells forming cribriform patterns at the periphery of solid tumor nests (Fig. 3, B). The epithelial cells as well as the dental papillalike ectomesenchymal cells of ameloblastic fibroma were diffusely positive to heparanase (Fig. 3, C) although the mesenchymal cells 


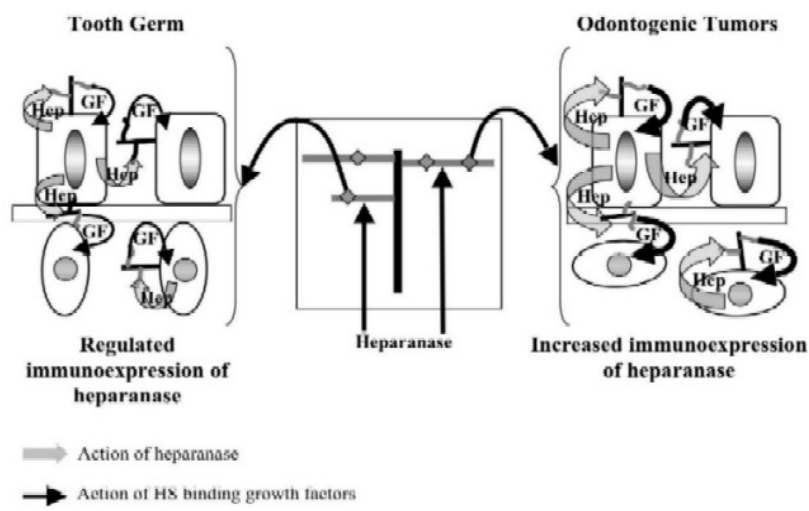

Figure 5. Role of heparanase in the release of HS binding growth factors in tooth germ and benign odontogenic tumors

Regulated expression and localization heparanase in tooth germ may result the physiologic growth and development of tooth germ by modulation of HS and control release of HS bound growth factors. Increased in immunoexpression of heparanase by the tumor cells may augment the release and action of HS bound growth factors which in turn are responsible for the development and progression of benign odontogenic tumors.

showed weaker intensity. Focal nuclear staining to heparanase was also observed in some mesenchymal cells (data not shown). In ameloblastoma, heparanase was primarily observed in peripheral epithelial cells of tumor nests and strands with stronger intensities at the budding areas of neoplastic epithelium and near
Tooth Germ


Ameloblastoma

(Follicular)
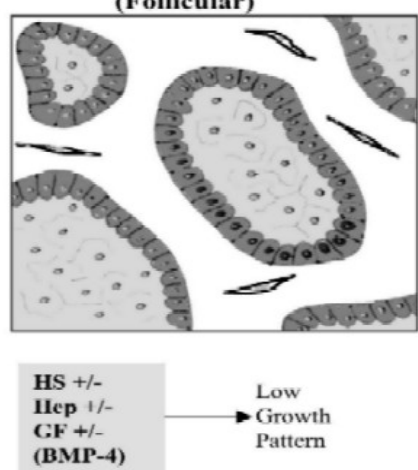

Figure 6. Schematic diagram showing the localization of HS, heparanase and BMP-4 in tooth germ and benign odontogenic tumors

In tooth germ (A), the growing cervical loops and the dental lamina showed co-localization of HS, heparanase and BMP-4. Benign epithelial odontogenic tumors (B, D) the neoplastic epithelial components showed positive immunoreaction to all proteins. In ameloblastic fibroma (C) the epithelial as well as the mesenchymal components were immunopositive. The difference in localization patterns of these molecules may be related to cellular differentiation and growth potential.
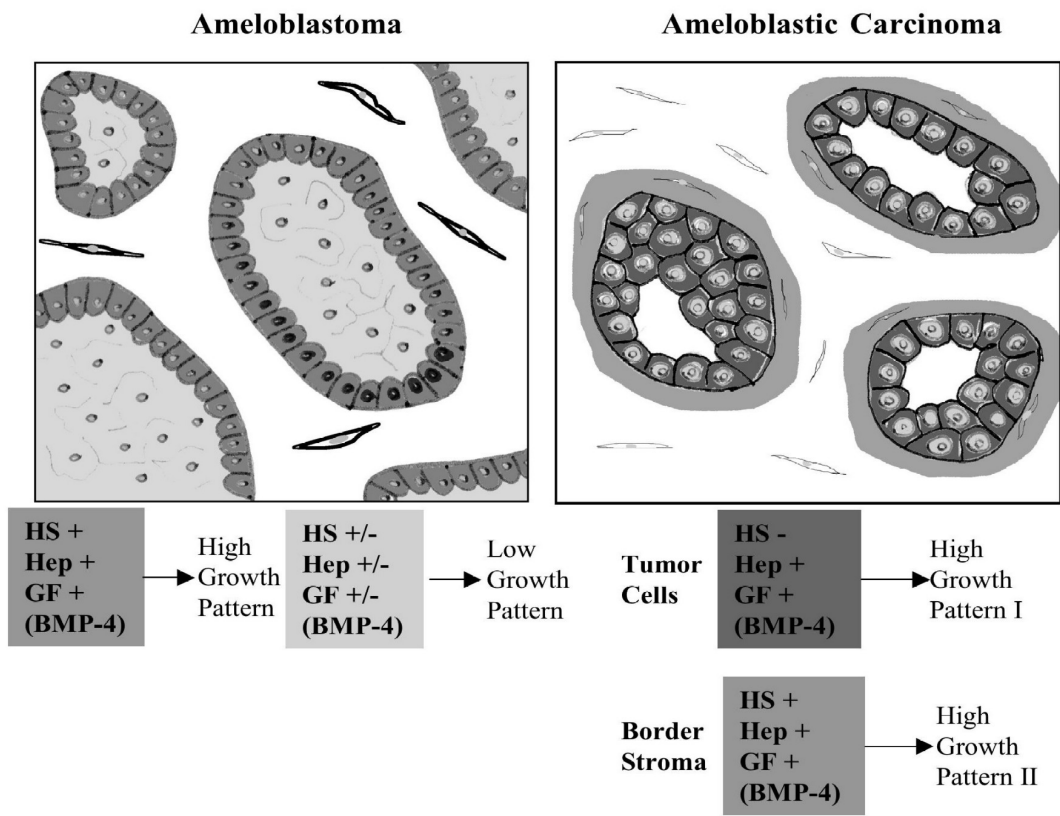

Figure 7 Schema illustrating localization of HS, Heparanase and BMP-4 in ameloblastoma and ameloblastic carcinoma

the invasive fronts (Fig. 3, D). Similar to HS, heparanase were not detected in stromal fibroblasts of ameloblastoma. The malignant epithelial nests of ameloblastic carcinoma showed very intense and diffuse intracellular staining to heparanase (Fig. 3, E) in contrast to the staining noted in ameloblastoma where heparanase was primarily localized in the basal cells.

Localization of BMP-4 (Fig. 4)

BMP-4 staining in the tooth germ was observed mainly in the 


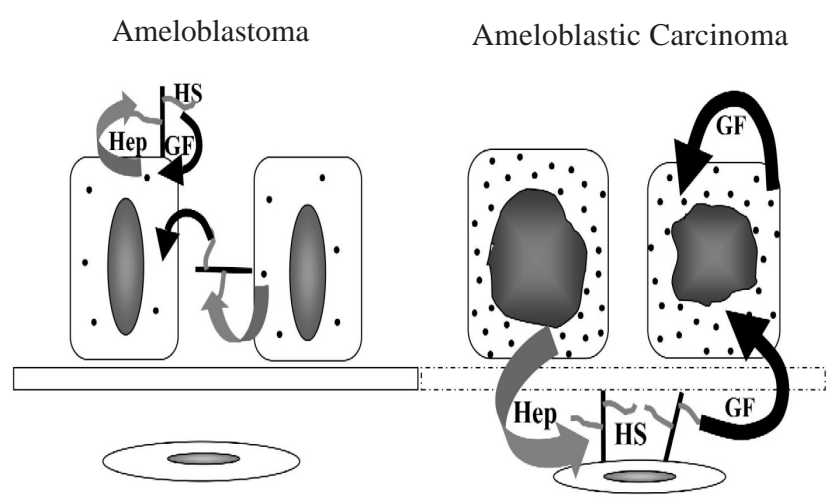

Heparanase produced by tumor cells on the HS molecules on the cell surface and release the tethered growth factors. Released growth factors in turn act on the tumor cell in the autocrine manner

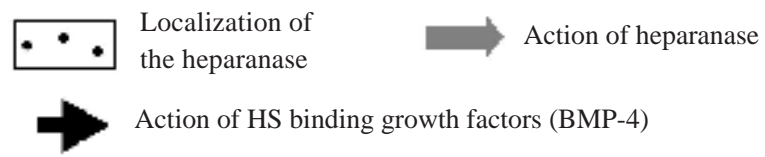

Figure 8 The suggestive functional roles of HS, heparanase and BMP-4 in relation to growth and progression of tumor cells in ameloblastoma and ameloblastic carcinoma

outer enamel epithelium and the growing cervical loops but not observed in other areas of enamel organs as well as in dental mesenchyme (Fig. 4, A). The expression pattern of BMP-4 in AOT was similar to that of heparanase but with a weaker intensity; diffuse cytoplasmic staining was observed in epithelial tumor cells with stronger staining in dark cuboidal cells forming cribriform patterns at the periphery of solid tumor nests (Fig. 4, B). In ameloblastic fibroma, BMP-4 was observed primarily in the peripheral columnar cells of the epithelial nests but only as limited focal staining in the mesenchymal components (Fig. 4, C). Similar to that of HS and heparanase, the immunoreaction to BMP-4 in ameloblastoma was localized predominantly in the peripheral columnar cells than the central stellate-like cells with no significant staining in the stromal fibroblasts (Fig. 4, D). As for ameloblastic carcinoma, BMP-4 was observed randomly in the cancer cells as well as in the stromal cells (Fig. 4, E).

\section{Discussion}

HSPG include a group of proteoglycans localized on the cell surfaces such as syndecans and glypicans and at the BMs and ECM such as perlecan, agrin and type XVIII collagen. Despite the tissue-specific expression of individual core protein, ligandbinding of HSPG depends on the structure of HS chains ${ }^{1}$. Although the precise functions of HS molecules in tooth development have not been clarified yet, one of their important functions may be the functional modulation of growth factors and morphogens with
Inter-relationship of Heparanase, Heparan Sulphate Bound Growth Factors and Cellular Sinalling System for Cell Proliferation and Differentiation

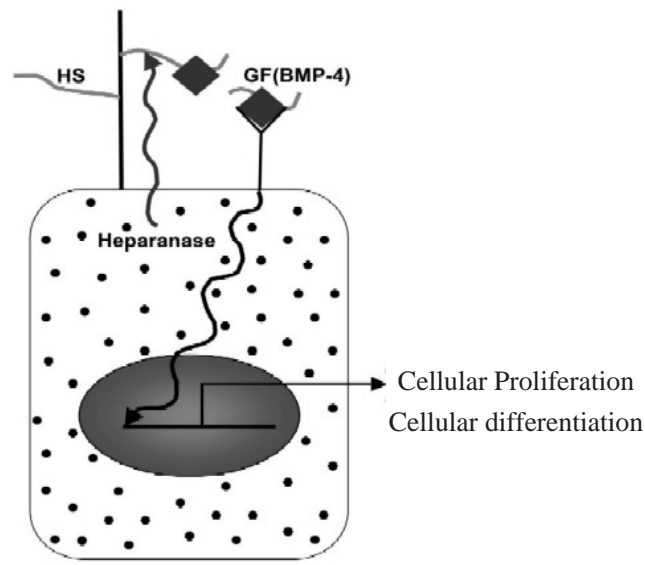

Figure 9The schematic diagram showing the role of heparanase, heparan sulphate and growth factors in relation to cellular proliferation and differentiation

their respective receptors.

The localization of HS in tooth germ with the strongest intensities in the stratum intermedium and dental papilla cells suggests that HS in general may have roles in cellular proliferation and differentiation through reciprocal and sequential signaling events. Heparanase overexpression has been reported to promote hair follicle morphogenesis and hair growth ${ }^{20}$. Since hair follicle morphogenesis and odontogenesis are both governed by similar growth factors and signaling pathways ${ }^{21)}$, heparanase expression in the outer and inner enamel epithelium of growing cervical loops suggests that it may be responsible for cellular growth through local modulation and release of HS bound growth factors. Moreover, previous reports have documented that nuclear heparanase in cancer cells is related to cellular differentiation ${ }^{22,23)}$. The nuclear staining to heparanase observed in some epithelial and mesenchymal cells in tooth germ may also correspond with cellular differentiation. Furthermore, nuclear staining to heparanase observed in tooth germ and ameloblastic fibroma implied that participation and interaction between epithelial and mesenchymal compartments might be important for the nuclear localization of heparanase.

The BMP family, which is part of the TGF- $\beta$ super family plays multiple roles in the differentiation of many tissues, and in the formation, development and patterning of several different organ systems including tooth germs. In the latter, BMP family acts as regulators in the sequential and reciprocal epithelial-mesenchymal interactions during tooth morphogenesis. These interactions in turn determine cell growth and differentiation in the developing tooth germ ${ }^{19}$, 24). BMP proteins are also known as HS bound molecules ${ }^{2)}$ and BMP-4 was used as an example of HS bound 
Han Phuu Pwint et al: Role of Heparanase in Odontogenic Tumors

growth factors in this study.

If for ameloblastoma alone, immunolocalization as well as expression of heparanase was relevant for the local invasion of tumor cells ${ }^{16)}$. But, the localization of heparanase in normal tooth germ and various odontogenic tumors ranging from hamartomatous lesion such as AOT to frankly malignant lesion such as ameloblastic carcinoma, suggested that heparanase may also be related to tumor growth possibly through local modulation of HS molecules and release of HS bound bioactive molecules. From the results, it was observed that HS, heparanase and BMP4 immunoexperession might correspond with the biological behavior of odontogenic tumors. Localization of BMP-4 in benign odontogenic tumors, more or less co-localized with HS and heparanase suggested that its localization, availability and function could be modulated by these molecules and thus affecting the growth and progression of tumor cells (Fig. 5).

In benign epithelial odontogenic tumors, the neoplastic epithelial components showed positive immunoreaction to HS, heparanase and BMP-4 but the mature fibrous stroma did not show significant staining. But in ameloblastic fibroma, the epithelial as well as the mesenchymal components of the tumor cells showed positive staining to all these molecules with BMP-4 the weakest intensity. Although all tumors are odontogenic in origin, tumor cells have different and unique biological behavior that will determine their fate and tumor progression. So, the difference in the localization patterns of HS and heparanase and BMP-4 molecules in benign odontogenic tumors may be related to the differentiation and the growth potential of tumor cells (Fig. 6). In general, the significant difference in immunoreaction between normal tooth germ and benign odontogenic tumors was the apparent increase in immunoexpression of heparanase in tumoral tissues especially by the neoplastic odontogenic epithelial cells.

Based on 2005 WHO histological typing of odontogenic tumors, all four cases studied belong to the category of ameloblastic carcinoma with overt cellular malignancy and aggressiveness. Localization of HS, heparanase and BMP-4 in ameloblastic carcinoma were distinct from that of ameloblastoma (Fig. 7). HS chains were strongly localized in the stroma of ameloblastic carcinoma in contrast to their confined localization in epithelial nests of ameloblastoma. Similar findings were reported in ovarian cancer, breast cancer and head and neck squamous cell carcinoma $25,26,27)$. It was suggested that loss or reduction of HS immunoexpression from cancer cells leads to decrease intercellular cohesion and increase potential for tumor invasion and metastasis. Moreover, reciprocal growth promoting loops may exist between cancer cells and stromal cells where in cancer cells produced certain cytokines thereby inducing stromal reaction. The activated stromal cells in turn produce HSPG to provide or modulate the activities of important growth factors for cancer cell proliferation and invasion ${ }^{25}$. Furthermore, a strong and diffuse immunoexpression of heparanase in all cancer cells of tumor nests was noted compared to the focally strong localization of heparanase in the peripheral cells of tumor nests in ameloblastoma. Diffuse immunoreaction to BMP-4 was noted in the cancer cells as well as the stromal cells of ameloblastic carcinoma with more random and weaker staining pattern than ameloblastoma. Taken together, the loss of HS and the increase in heparanase may indicate the malignant transformation of ameloblastoma to ameloblastic carcinoma. It was suggested that HS bound growth factors; BMP4 protein in this case, might function in autocrine and paracrine manners among the cancer cells and the stromal cells promoting growth, proliferation and progression of cancer cells while they function in autocrine manners within the tumor cells of ameloblastoma (Fig. 8). This may also differentiate benign and malignant forms of ameloblastoma.

Interestingly, the BMs surrounding the epithelial tumor nests of benign tumors were well-defined despite the strong localization of heparanase. However, BMs surrounding the cancer nests in ameloblastic carcinoma were irregular and unclear. These suggest that heparanase in benign tumors may be tightly and locally regulated and related to local invasion. On the contrary, heparanase was clearly related to disseminated invasion in ameloblastic carcinoma.

In summary, this study demonstrated the general distribution patterns of HS and importantly the localization of heparanase in normal human tooth germ and in odontogenic tumors. In physiological condition, HS, heparanase and BMP-4 may be temporally regulated in relation to cellular growth and function (Fig. 9). Heparanase may have physiologic function in tooth germ and the increase in immunoexpression may be an initiating factor for the neoplastic transformation of odontogenic tissues. The stromal localization of HS and BMP-4 together with apparent increase in immunoexpression of heparanase in cancer cells may represent the malignant progression of ameloblastoma to ameloblastic carcinoma. From this study, it can be concluded that the growth and progression of benign and malignant odontogenic tumors are related to the deranged immunoexpression and localization of HS and heparanase molecules and that heparanase may be responsible for growth and progression of odontogenic tumors by modulating the availability and function of HS binding growth factors. Further molecular and biochemical studies are necessary to determine the detailed functional mechanisms of HS and heparanase in odontogenesis and odontogenic tumor progression.

\section{Acknowledgement}

This work was supported by grants-in-aid for scientific research (17406027) from the Japanese Ministry of Education, Culture, Sports, Science and Technology and grants-in-aid (103971370992) from Astrazeneca Foundation (To M.G).

\section{References}


1. Esko JD and Selleck SB. Order out of chaos: assembly of ligand binding sites in heparan sulfate. Annu Rev Biochem 71: 435-471, 2002

2. Bernfield M, Gotte M, Park PW, Reizes O, Fitzgerald ML, Lincecum J and Zako M. Functions of cell surface heparan sulfate proteoglycans. Annu Rev Biochem 68: 729-777; 1999

3. Blackhall FH, Merry CLR, Davies EJ and Jayson GC. Heparan sulfate proteoglycans and cancer. B J Cancer 85(8): 10941098, 2001

4. Bame KJ. Heparanases: endoglycosidases that degrade heparan sulfate proteoglycans. Glycobiology 11(6): 91R-98R, 2001

5. Vlodavsky I, Goldshmidt O, Zcharia E, Atzmon R, RanginiGuatta Z, Elkin M, Pertz T and Friedmann P. Mammalian heparanase: involvement in cancer metastasis, angiogenesis and normal development. Semin Cancer Biol 12(2): 121-129; 2002

6. Goldshmidt O, Zcharia E, Cohen M, Aingorn H, Cohen I, Nadav L, Katz BZ, Geiger B, Vlodavsky I. Heparanase mediates cell adhesion independent of its enzymatic activity. FASEB J 17(9): 1015-1025, 2003

7. Imada T, Matsuoka J, Nobuhisa T, Okawa T, Murata T, Tabuchi Y, Shirakawa Y, Ohara N, Gunduz M, Nagatsuka H et al. COX2 induction by heparanase in the progression of breast cancer. Int J Mol Med 17(2):221-228, 2006

8. Zetser A, Bashenko Y, Edovitsky E, Levy-Adam F, Vlodavsky I, Ilan N. Heparanase induces vascular endothelial growth factor expression: correlation with p38 phosphorylation levels and Src activation. Cancer Res 66(3): 1455-1463, 2006

9. Thesleff I, Partanen AM, Vainio S. Epithelial-mesenchymal interactions in tooth morphogenesis: the roles of extracellular matrix, growth factors and cell surface receptors. J Craniofac Genet Dev Biol 11(4): 229-237, 1991

10. Zhang YD, Chen Z, Song YQ, LIU C, Chen YP. Making a tooth: growth factors, transcription factors and stem cells. Cell Res 15(5): 301-316, 2005

11. Barnes L, Eveson JW, Reichart P, Sidransky D (Eds) World Health Organization Classification of Tumors. Pathology and Genetics of Head and Neck tumors. IARC press: Lyon, 283327, 2005

12. Bai XM, Van der Schueren B, Cassiman JJ, Van den Berghe H, David G. Differential expression of multiple cell-surface heparan sulfate proteoglycans during embryonic tooth development. J Histochem Cytochem 42(8): 1043-1054, 1994

13. Ida-Yonemochi H, Ohshiro K, Swelam W, Metwaly H, Saku T. Perlecan, a basement membrane type heparan sulfate proteoglycan (perlecan) in ameloblastomas: its intercellular localization in stellate reticulum-like foci and biosynthesis by tumor cells in culture. Virchows Arch 441(2): 165-173, 2002

14. Murata M, Cheng J, Horino K, Hara K, Shimokawa H, Saku T. Enamel proteins and extracellular matrix molecules are colocalized in the pseudocystic stromal space of adenomatoic odontogenic tumor. J Oral Pathol Med 29(10): 483-490; 2000.

15. Kumamoto H. Molecular pathology of odontogenic tumors. J Oral Pathol Med 35: 65-74, 2006

16. Nagatsuka H, Han PP, Tsujigiwa H, Siar CH, Gunduz M, Sugahara $\mathrm{T}$ et al. Heparanase gene and protein expression in ameloblastoma: possible role in local invasion of tumor cells. Oral Oncol 41(5): 542-548, 2005

17. Vainio S, Karavanova I, Jowett A, Thesleff I. Identification of BMP-4 as a signal mediating secondary induction between epithelial and mesenchymal tissues during early tooth development. Cell 75(1): 45-58, 1993

18. Kumamoto H, Ooya K. Expression of bone morphogenetic proteins and their associated molecules in ameloblastoma and adenomatoid odontogenic tumors. Oral Diseases 12(2): 16370, 2006

19. van den Born J, Salmivirta K, Henttinen T, Ostman N, Ishimaru T, Miyaura S, Yoshida K, Salmivirta M. Novel heparan sulfate structures revealed by monoclonal antibodies. J Biol Chem 280(21): 20516-20523, 2005

20. Zcharia E, Matzger S, Chajek-Shaul T, Aingorn H, Elkin M, Friedmann $\mathrm{Y}$ et al. Transgenic expression of mammalian heparanase uncovers physiological functions of heparan sulfate in tissue morphogenesis, vascularization, and feeding behavior. FASEB J 18(2): 252-263, 2004

21. Thesleff I, Vaahtokari A and Partanen AM. Regulation of organogenesis: common molecular mechanism regulating the development of teeth and other organs. Int J Dev Biol 39:3550, 1995

22. Ohkawa T, Naomoto Y, Takaoka M, Gunduz M, Tsujigiwa H, Nagatsuka H et al. Localization of heparanase in esophageal cancer cells: respective roles in prognosis and differentiation. Lab Invest 84(10): 1289-1304, 2004

23. Nobuhisa T, Naomoto Y, Takaoka M, Tabuchi Y, Gunduz M, Nagatsuka $\mathrm{H}$ et al. Emergence of nuclear heparanase induces differentiation of human mammary cancer cells. Bochem Biophys Res Commun 331(1): 175-180, 2005

24. Heikinheimo K. Stage-specific expression of decapentaplegicVg-related genes 2, 4, and 6 (bone morphogenetic proteins 2, 4 , and 6) during human tooth morphogenesis. J Dent Res 73:590-7, 1994

25. Davies EJ, Blackhall FH, Shanks JH, David G, McGown AT, Swindell R et al. Distribution and clinical significance of heparan sulfate proteoglycans in ovarian cancer. Clin Cancer Res 10(15): 5178-5186, 2004

26. Maeda T, Alexander CM, Friedl A. Induction of syndecans-1 expression in stromal fibroblasts promotes proliferation of human breast cancer cells. Cancer Res 64(2): 612-621, 2004

27. Mukunyadzi P, Liu K, Hanna EY, Suen JY, Fan CY. Induced expression of syndecans-1 in the stroma of head and neck squamous cell carcinoma. Mod Pathol 16(8): 796-801, 2003 